\title{
Completion of binary statements by children at three academic levels
}

\author{
BARBARA HUNT LAZERSON and EUGENE IRVING \\ Department of Curriculum and Instruction, Illinois State University, Normal, Mlinois 61761
}

\begin{abstract}
This study was designed to examine the strategy employed by school-aged subjects when completing binary statements. Thirty-two incomplete binary statements representing four statement conditions (true affirmative, true negative, false affirmative, and false negative) were administered to 120 children randomly selected from three academic levels. A response-index change model is postulated as representing the strategy employed by these school-aged subjects when completing binary statements. According to this model, the factor that causes response time to differ across the four statement conditions is the number of times the child is required to change the set of his response index. The child begins the task of completing a binary statement by reading and encoding the incomplete statement. He then selects the numeral that corresponds to the adjective "odd" or "even" contained in the predicate of the incomplete statement. The child's response index is now set to respond with this numeral. What the child does at this point will depend upon the type of statement condition with which he is working. If the child is completing a TA statement, no response-index change is required. He simply gives as his answer the numeral to which his response index is set. If the child is completing an FA statement or a TN statement, one response-index change is required. If the child is completing an FN statement, two response-index changes are required.
\end{abstract}

In the years following publication of Chomsky's Syntactic Structures (1957), a number of studies were conducted in an effort to determine the influence of transformations upon sentence comprehension (e.g., Gough, 1965; Slobin, 1966; Wason, 1961). In such studies, the hypothesis being tested was that the ease with which an individual comprehends a sentence is "a function of the number and nature of the transformations separating it from its underlying structure" (Gough, 1966, p. 492). From a psycholinguistic perspective, these studies have been disappointing, for the relative difficulty of comprehending various types of sentences has been found to have little to do with linguistic factors per se. Performance on sentence verification tasks is more appropriately explained as a function of the problem solving strategies employed by the subjects during the experiment (Glucksberg \& Danks, 1975 , p. 101). More specifically, performance on sentence verification tasks can be accounted for on the basis of the number of elementary mental operations involved (Carpenter \& Just, 1975; Clark \& Chase, 1972; Just, 1974; Lazerson \& Irving, 1976; Trabasso, Rollins, \& Shaughnessy, 1971).

Carpenter and Just (1975) have developed a model that parsimoniously accounts for the results obtained in sentence comprehension experiments requiring the

This study was supported by a grant from the Graduate Office of Illinois State University. The authors wish to thank Linda Florek Failla for collecting and recording the data. Requests for reprints should be sent to Barbara Lazerson, Department of Curriculum and Instruction, Illinois State University, Normal, Illinois 61761 . subject to determine whether a sentence is true or false. Their constituent comparison model accounts for verification latencies for a variety of sentences on the basis of a single underlying iterative operation, the time required to find and compare one pair of constituents. The model postulates that verification latency will increase linearly with an increase in the number of hypothesized constituent comparisons.

Lazerson and Irving (1976) found that the constituent comparison model accounted for the performance of children on semantic memory verification tasks involving binary statements. That is, the linear increase in verification latencies across the four statement conditions (true affirmative, false affirmative, false negative, true negative) corresponded to the number of hypothesized constituent comparisons. This suggests that the children employed in the semantic memory verification study verified sentences by finding and comparing, pair by pair, the corresponding constituents from the printed sentence representation and the semantic memory representation.

What strategy do children utilize when the task is completion of binary statements rather than verification of binary statements? An examination of the literature shows that the completion of binary statements has been largely ignored by researchers. As far as the authors know, only Wason (1961) has done research involving the completion of binary statements-and his work was with adult subjects. It was the purpose of the present study to examine the strategy employed by school-aged subjects in completing binary statements. The sample was selected in such a manner that the results of the study could be compared with the results 
of the authors' previous research. However, the results of this study are not comparable with the results obtained in Wason's study, because Wason employed a slightly different method. ${ }^{1}$

\section{METHOD}

\section{Subjects}

The subjects were 120 children, randomly selected from the pupil population of Illinois State University's Metcalf Laboratory School. An equal number of children (40) were selected from each of three academic levels: Grades 1 through 3 (Academic Level $\mathrm{C}_{1}$ ), Grades 4 through 6 (Academic Level $\mathrm{C}_{2}$ ), and Grades 7 and 8 (Academic Level $C_{3}$ ). The mean ages of the three groups were 8 years, 8 months; 10 years, 6 months; and 13 years, 1 month, respectively. The median IQ of the population from which the sample was selected was 111. The socioeconomic composition of the group was predominantly white upper middle-class professional.

A stratified constant sample (i.e., an equal number of maies and females and an equal number of children above and below the median IQ) was employed, since sex and IQ are variables that might have influenced the results.

\section{Screening Procedures}

In order to participate in the research, each child had to demonstrate his ability (1) to recognize visually the numerals 1 through 10 inclusive; (2) to associate correctly the concept odd-even to the numerals; (3) to understand the concept of truth-falsity; (4) to read and comprehend the incomplete statement conditions; and, (5) to complete orally the statement conditions.

\section{Practice Trials}

The numerals 1 and 2 were used to develop binarycompletion statements that varied in terms of the affirmativenegative dimension. A total of four practice completion statements (one numeral pair by two odd by two even) as shown in Figure 1 were printed on $3 \times 5$ in. cards. The four practice cards were presented randomly to each child twice, once before the child was asked to produce true statements and once before he was asked to produce false statements. The child was instructed to produce true statements on those practice trials preceding the experimental trials in which he was expected to produce true statements and to produce false statements on those practice trials preceding the experimental trials in which he was expected to produce false statements.

\section{Experimental Trials}

The numerals 3 through 10 inclusive were divided into the following pairs: 3,$4 ; 5,6 ; 7,8 ; 9,10$. Four binary-completion statements similar to those in Figure 1 were developed for each pair of numerals. The total number of statement cards used for the experimental trials was 16 (four numeral pairs by two odd by two even). Two sets of cards were developed, one set with the odd numeral first and one set with the even numeral first. Each set of cards was used with half of the subjects. This procedure was employed in order to determine whether the ordering of the numerals at the top of the card had a significant influence on the performance of the subjects.

Four statement conditions were used in the study: true affirmative (TA), true negative (TN), false affirmative (FA), and false negative (FN). Consequently, each subject completed a total of 32 binary statements (8 of each statement condition type). The affirmative-negative dimension was varied by including or eliminating the word "not," as shown in Figure 1. The truth-falsity dimension was varied by instructions. The child was asked to produce false statements during one presentation block of the 16 statement cards and true statements

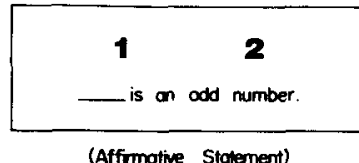

(Afimative Stotement)

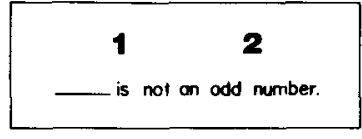

(Negative Statement)

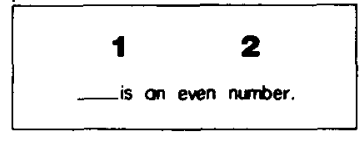

(Affimative Statement)

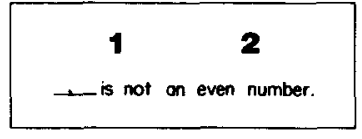

(Negative Stutement)
Figure 1. Examples of the binary-completion statement cards used in this study.

during the other presentation block of the same 16 statement cards. Half of the subjects were asked to produce true statements on the first presentation and false statements on the second presentation. The other half of the subjects were given the opposite sequence.

The experimenter worked with each child individually. The child's responses were recorded on a cassette tape recorder. The order in which the experimental items were presented was randomized for each of the subjects. When the child had completed all of the experimental items, he was asked to respond to the question, "How did you figure out what numeral to put in the blank?"

\section{Measurement of Variables}

The dependent variables in the study were errors and response time for correct items. The error count was tabulated for each child by counting the number of instances in which he responded with the wrong numeral. The response time for correct iterns was measured by means of a stopwatch by recording the lapsed time between the examiner's presentation of the card to the child, which was signaled on the tape by the examiner's statement "next," and the child's utterance of the numeral that constituted his response. All of the response time latencies were measured by a single judge. A split-half reliability coefficient of +.92 was obtained as a measure of the consistency of the scoring by that judge.

\section{RESULTS}

Analysis of variance tests indicated that neither the number of errors $[F(1,114)=.18, p>.20]$ nor response time $[F(1,114)=.05, p>.20]$ was influenced significantly by the order of the numerals at the top of the card. Likewise, neither the number of errors $[F(1,114)=.69, p>.20]$ nor response time $[F(1,114)=1.59, p>.20]$ was influenced significantly by whether the subjects produced true statements first or false statements first.

Both response time data and error data were analyzed via a 3 (academic level) by 4 (statement condition) repeated measures ANOVA. The response time ANOVA yielded significant results for both academic level $[F(2,117)=23.04, p<.001]$ and statement condition $[F(3,351)=37.72, p<.001]$. Interaction of Statement Condition by Academic Level was not significant $[F(6,351)=.89, p>.20]$. Post hoc comparisons among academic level means indicated that Academic Level $C_{1}$ required a significantly greater response time than 
Table 1

Mean Response Time in Seconds for Correct Responses

\begin{tabular}{lrrrr}
\hline & \multicolumn{4}{c}{ Statement Condition } \\
\cline { 2 - 5 } Academic Level & TA & TN & FA & FN \\
\hline $\mathrm{C}_{1}$ & 3.7 & 4.8 & 4.9 & 5.7 \\
$\mathrm{C}_{2}$ & 2.3 & 3.2 & 3.1 & 3.8 \\
$\mathrm{C}_{3}$ & 1.8 & 2.4 & 2.4 & 3.0 \\
Mean & 2.6 & 3.5 & 3.5 & 4.2 \\
Standard Error & .1 & .2 & .2 & .2 \\
\hline
\end{tabular}

did Academic Level $C_{3}$. However, there was no significant difference in response time between either Academic Levels $C_{1}$ and $C_{2}$ or Academic Levels $C_{2}$ and $\mathrm{C}_{3}$. The error ANOVA yielded significant results for statement condition only $[\mathrm{F}(3,351)=12.14, \mathrm{p}<.001]$. Neither academic level $[F(2,117)=2.19, p>10]$ nor the interaction of Statement Condition by Academic Level $[F(6,351)=1.39, p>.20]$ was significant. These results suggest that developmental differences across academic levels were minimal. The performance of all subjects, regardless of academic level, was primarily a function of the number of response-index changes (discussed below) required to complete the binary statements employed in the study. However, the oldest subjects (Academic Level $\mathrm{C}_{3}$ ) were able to complete such statements in significantly less time than the youngest subjects (Academic Level $\mathrm{C}_{1}$ ).

Mean response times, rounded to the nearest $.10 \mathrm{sec}$, for correct responses distributed according to statement condition and academic level are shown in Table 1. As mentioned above, a significant $F$ value was obtained for statement conditions where response time was concerned. Post hoc comparisons among statement condition means indicated (1) that FN statements required a significantly greater time for completion than did any of the other statement conditions, (2) that TN and FA statements required statistically equivalent amounts of time for completion, and (3) that TA statements required significantly less time for completion than all of the other statement conditions.

Table 2 shows the number of errors distributed according to statement condition and academic level. Where errors were concerned, a significant $F$ value was obtained for statement condition only, as mentioned above. Post hoc comparisons among statement condition means indicated (1) that a significantly greater number of errors were made for FN statements than for any other statement condition, (2) that the number of errors made on FA statements was not significantly greater than the number of errors made on TA statements, but (3) that the total errors for TN statements fell nine short of being significantly greater than total errors for TA statements.

The response-index change model depicted in Figure 2 accounts for the results obtained in this study. According to this model, the subject first reads and encodes the incomplete binary statement. He then examines the numerals at the top of the card and selects one as corresponding to the adjective "odd" or "even" in the predicate of the incomplete statement. The subject's response index is now set to respond with this numeral. If the subject is completing a TA statement, no response-index change is required. If any of the other statement conditions is being completed, the subject must change the set of his response index at least once. In the case of a TN incomplete binary statement, the subject must react to the presence of the word "not" in the statement as a command to change the response index to the other numeral before stating his answer. ${ }^{2}$ TN statements thus require a single response-index change. When the subject has been instructed to construct false binary statements, that instruction constitutes a command to change the response index to the other numeral before responding to an affirmative statement. Therefore, FA statements also require a single responseindex change. FN statements require not only the change necessitated by the instruction to construct false binary statements; they also require the change necessitated by the presence of the word "not" in the incomplete binary statement. FN statements thus require two response-index changes.

The pattern of significant differences for response time data, which may be symbolized as follows, is precisely that predicted by the response-index change model: $\mathrm{TA}<\mathrm{TN}=\mathbf{F A}<\mathrm{FN}$. As Table 1 shows, mean response times, rounded to the nearest $.10 \mathrm{sec}$, for TN and FA statements are equal $(3.5 \mathrm{sec})$, as this model predicts. The response-index change model also predicts that the difference between TN - TA response time means would be statistically equivalent to the difference between FN - FA response time means, because TN statements require one more response-index change than TA statements and FN statements require one more response-index change than FA statements. A $t$ test for related measures was employed to determine whether this was the case. The results were found to be nonsignificant $[t(119)=.16, p>.20]$. This suggests that the $.9-\mathrm{sec}$ difference between $2.6 \mathrm{sec}$ (the TA mean) and $3.5 \mathrm{sec}$ (the TN mean) is not significantly greater than the .7-sec difference between $3.5 \mathrm{sec}$ (the FA mean) and $4.2 \mathrm{sec}$ (the FN mean).

At first glance, the error data appear to be less

Table 2

Total Errors

\begin{tabular}{cccccc}
\hline & \multicolumn{5}{c}{ Statement Condition } \\
\cline { 2 - 6 } Academic Level & TA & TN & FA & FN & Total \\
\hline $\mathrm{C}_{1}$ & 28 & 31 & 46 & 72 & 177 \\
$\mathrm{C}_{2}$ & 18 & 27 & 34 & 39 & 118 \\
$\mathrm{C}_{3}$ & 13 & 41 & 33 & 53 & 140 \\
Total & 59 & 99 & 113 & 164 & 435 \\
\hline
\end{tabular}




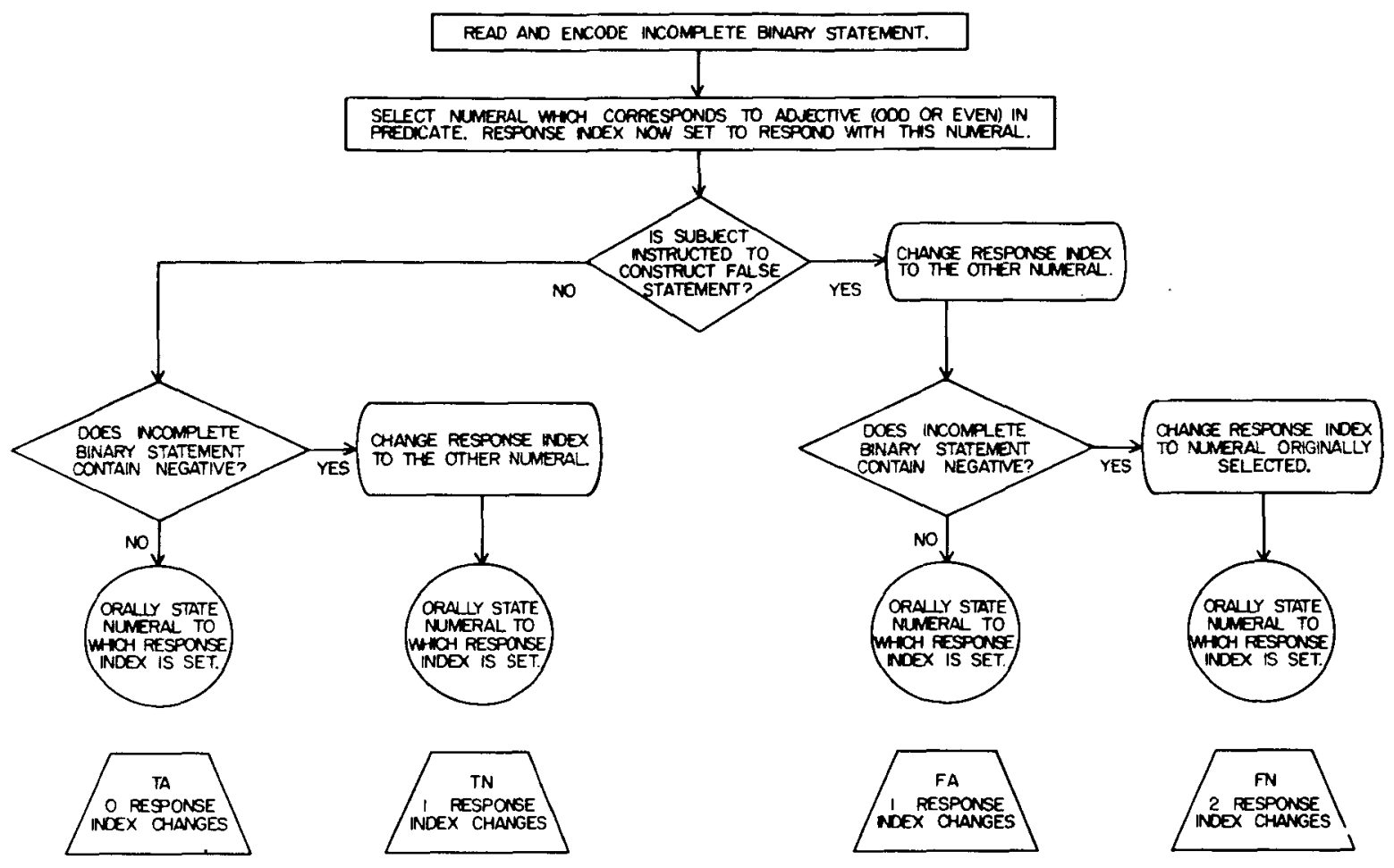

Figure 2. Response-index change model.

supportive of the response-index change model than do the response time data. But, upon closer examination, this is found not to be so. The semirandom nature of the error pattern for the four statement conditions across the three academic levels might be partly a function of the low observation rates in each of the cells (320 trials per cell), since total error rate, which was 960 trials per cell, more closely approximates the pattern predicted by the model. In fact, the pattern for total errors falls only nine short of fitting the predicted pattern; for had the total errors for TN statements been 108 rather than 99 (in a cell where the total number of errors could have fallen anywhere between 0 and 960 inclusive), total errors for TN statements would have been significantly greater than total errors for TA statements and the pattern of significant differences for total errors would replicate the pattern of significant differences for response time data.

The fact that the pattern of significant differences for total errors does not replicate, but only approximates, the pattern of significant differences for response time data does not undermine the model described in this study, for the response-index change model is primarily a time-based model and only secondarily an error-based model. That is, each response-index change provides the opportunity for, but does not require, the commission of an error. However, each response-index change requires the consumption of an amount of time. Furthermore, errors are not cumulative for a single trial, but response time is cumulative. In other words, a subject's answer is an all-or-none response that is either right or wrong. When these points are taken into account, it can be seen that the pattern of significant differences for total errors parallels the pattern of significant differences for response time quite well.

An examination of the responses of the subjects to the question "How did you figure out what numeral to put in the blank?" lends support to the responseindex change model. Some children, of course, either had no insights whatever into the procedure they employed in completing the binary statements used in the study or were unable to explain the procedure employed. However, some of the children were more aware of the strategy they used. Although no child answered the preceding question by describing the entire response-index change model shown in Figure 2, several of them described at least one stage of the model, as the following statements show: (Number 49) "You know, I just looked at a sentence, then the numbers. Because like if it said 'blank is an odd number,' I picked the odd number instead of just going 5 and 6 and what is an odd number. It's faster." (Number 60) "If you tell me to make them false, like the even number would be odd and the odd would be you know, like even. And if you told me to give you a true answer, well the odd would be odd 
and the even would be even." (Number 79) "You look at the statement to see if it says 'not' and then you put the opposite in. You have to look to see if it says 'not."'

The question of whether sex influenced performance on the binary statements employed in the study was examined by means of two 2 (sex) by 4 (statement condition) repeated measures ANOVAs, one for errors and one for response time. Neither errors $[F(1,118)=.38, \quad p>.20]$ nor response time $[F(1,118)=.70, p>.20]$ was significantly influenced by sex. Furthermore, interaction effects for both errors $[F(3,354)=.51, p>.20]$ and response time $[\mathrm{F}(3,354)=.26, \mathrm{p}>.20]$ were nonsignificant. These results suggest that the performance of both males and females was primarily a function of the number of response-index changes required to complete the binary statements employed in the study.

The question of whether IQ level influenced performance on the binary statements employed in the study was examined by means of two 2 (IQ level) by 4 (statement condition) ANOVAs, one for errors and one for response time. It was found that below-median IQ subjects, in contrast to above-median IQ subjects, made significantly more errors $[F(1,118)=14.16$, $\mathrm{p}<.001]$ and required a significantly greater response time $[F(1,118)=11.06, \quad p<.005] . \quad$ However, interaction effects for both errors $[F(3,354)=.51$, $\mathrm{p}>.20]$ and response time $[\mathrm{F}(3,354)=1.53, \mathrm{p}>.20]$ were nonsignificant. These results suggest that the performance of both above-median IQ subjects and below-median IQ subjects was primarily a function of the number of response-index changes required to complete the binary statements, but that the task of completing such statements was more difficult, as measured by errors and response time, for the belowmedian IQ group than for the above-median IQ group.

To summarize, the number of response-index changes required to complete binary statements was the overriding factor influencing subject performance. The factor cut across the subject variables of sex, academic level, and IQ level. Subject variables, on the other hand, differed with regard to influence upon performance. Since male subjects and female subjects did not differ significantly with regard to either response time or errors, sex may be viewed as playing an insignificant role in the completion of binary statements. The influence of academic level was minimal. Of the three response time comparisons between academic levels and the three error comparisons between academic levels, only one was significant. That is, subjects at Academic Level $C_{1}$ required a significantly greater amount of time to complete binary statements than did subjects at Academic Level $C_{3}$. IQ level was the only subject variable that significantly influenced both measures of performance. Below-median IQ subjects, in contrast to above-median IQ subjects, required significantly greater response time and made a significantly greater number of errors.

\section{DISCUSSION}

Two major points will be made in the discussion. First, the problem solving strategy employed by schoolaged subjects when completing binary statements is different from the problem solving strategy employed by school-aged subjects when verifying binary statements via semantic memory. Second, both the response-index change model and the constituent comparison model attempt to account for the strategies employed by the subjects on the basis of a single, iterative operation.

When the task is one of completing a binary statement by selecting one of two numerals listed above the statement, the number of response-index changes required to perform the task correctly is the mental operation that causes response time to differ across statement conditions. The response-index change model has already been described. When the task is one of verifying statements via semantic memory, the number of constituent comparisons required to verify a statement is the mental operation that causes response time to differ across statement conditions (Lazerson \& Irving, 1976). For purposes of comparison, the constituent comparison model is briefly described below.

The constituent comparison model developed by Carpenter and Just (1975) accounts for the results obtained in sentence verification experiments on the basis of a single underlying iterative operation, finding and comparing, pair by pair, the corresponding constituents from the printed sentence representation and the semantic memory representation. This model postulates that, after the subject has read and encoded the printed sentence, he has the following items in his memory: (1) a printed sentence representation; (2) a semantic memory representation, which is the form in which the subject's knowledge of the oddness or evenness of numerals is stored; and (3) a response index set to "true." At this point, the subject is ready to begin the process of comparing, pair by pair, the constituents found in the printed sentence representation with those found in the semantic memory representation. Constituent comparisons are of two types, inner proposition comparisons and polarity marker comparisons. The former involve a comparison of the lexical item "odd" or "even" found in the printed statement with the knowledge of the oddness or evenness of numerals stored in the subject's semantic memory. The latter involve a comparison of the affirmative-negative dimension of the printed statement with the affirmative nature of the knowledge of numerals stored in the subject's semantic memory. Comparisons proceed until a mismatch is found between the printed statement representation and the semantic 
memory representation. At this point, the response index is changed, the mismatch is tagged, and the comparison process is reinitialized. Mean response time for the four statement conditions (TA, FA, FN, TN) is a function of the number of constituent comparisons that must be made in order to ascertain whether a statement is true or false.

Both the response-index change model and the constituent comparison model are primarily time-based models and only secondarily error-based models. Consequently, three basic assumptions underlie both of the models. The first assumption is that, while all aspects of the problem solving situation, such as reading and encoding statements, are time consuming, these tasks are consistent across all statement conditions and do not, therefore, contribute significantly to the response time differential across statement conditions. The second assumption is that a single iterative operation, which is a function of the problem solving strategy employed by the subjects, accounts for the response time differential across statement conditions. That is, every repetition of the iterative operation requires the consumption of an equal amount of time that is cumulative for a given statement condition. The third assumption is that every repetition of the iterative operation postulated by each of the models provides the opportunity for, but does not require, the commission of an error.

\section{REFERENCES}

CARPenter, P., \& Just, M. A. Sentence comprehension: A psycholinguistic processing model of verification. Psychological Review, 1975, 82, 45-73.

CHomsky, N. Syntactic structures. The Hague: Mouton, 1957.

Clark, H., \& Chase, W. On the process of comparing sentences against pictures. Cognitive Psychology, 1972, 3, $472-517$.

GLUCKSBERG, S., \& DANKs, J. H. Experimental psycholinguistics: An introduction. Hillsdale, N.J: Lawrence Erlbaum, 1975.
GovGH, P. Grammatical transformations and speed of understanding. Joumal of Verbal Learning and Verbal Behavior, 1965, 4, 107-111.

Gough, P. The verification of sentences: The effects of delay of evidence and sentence length. Journal of Verbal Learning and Verbal Behavior, 1966, 5, 492.496.

Just, M. A. Comprehending quantified sentences: The relation between sentence-picture and semantic memory verification. Cognitive Psychology, 1974, 6, 216-236.

LAzerson, B. H., \& IRVING, E. Semantic memory verification of binary statements by children at three academic levels. Journal of Experimental Child Psychology, 1976, 22, 143-154.

SLOBIN, D. Grammatical transformations and sentence comprehension in childhood and adulthood. Journal of Verbal Learning and Verbal Behavior, 1966, 5, 219-227.

Trabasso, T., Rollins, H., \& Shaughnessy, E. Storage and verification stages in processing concepts. Cognitive Psychology, 1971, 2, 239-289.

WAson, P. C. Response to affirmative and negative binary statements. British Journal of Psychology, 1961, 52, 133-142.

\section{NOTES}

1. Wason (1961, p. 134) had the following incomplete statements printed on cards: "... is an even number," "... is an odd number," "... is not an even number," "... is not an odd number." The digits 2 to 9 were stencilled on a display card that remained in front of the subject throughout the completion portion of the experiment. Before presenting each statement, an oral instruction was given to the subject to make the statement either true or false.

2. A basic assumption of the response-index change model is that reading-encoding time is not significantly increased by the presence of the word "not" in the incomplete binary statement. Two features of the response time data lend at least indirect support to this assumption. First, there was no significant difference between the mean response time for TN statements, which contain the word "not," and FA statements, which lack the word "not," at any of the three academic levels. Second, at Academic Levels $C_{1}$ and $C_{3}$ the statement condition containing the word "not" had a slightly smaller mean response time than the statement condition lacking the word "not."

(Received for publication August 24, 1976; revision accepted January $12,1977$. 